\title{
LOMA L'ÁNTIGO: CONSIDERACIONES SOBRE LA ARQUITECTURA DE UN POBLADO TARDÍO EN EL VALLE DEL CAJÓN (CATAMARCA, ARGENTINA)
}

\author{
María Fabiana Bugliani \\ CONICET-Museo Etnográfico. Moreno 350, Ciudad Autónoma de Buenos Aires. E-mail: fbugliani@gmail.com
}

Presentado el: 29/11/2011 - Aceptado 20/02/2012

\begin{abstract}
Resumen
A partir de las nuevas investigaciones iniciadas en el sitio Loma l'Ántigo, recientemente se ha renovado el interés en los estudios del Período Tardío en el valle del Cajón. El relevamiento arquitectónico del asentamiento ha permitido conocer la distribución y articulación de estructuras y rasgos construidos, las características del conjunto arquitectónico y las técnicas de construcción implementadas. Se reúnen los resultados de tal estudio y se provee una primera aproximación sobre la organización del espacio en el asentamiento y las modalidades estilísticas del conjunto arquitectónico. Loma l'Ántigo se presenta como un poblado aglomerado asentado en la cima de un morro que determina su configuración interna, al tiempo que lo emplaza en un sitio destacado en el paisaje.
\end{abstract}

Palabras claves: Loma l'Ántigo, arquitectura, asentamiento, Período Tardío.

\begin{abstract}
Recently, the interest in the studies of the Late Period in the Cajon valley has been renewed from the new investigations initiated in the site Loma l'Ántigo. The architectural survey of the settlement has allowed understanding the distribution and articulation of structures and constructed features, the characteristics of the architectural ensemble and construction techniques implemented. They meet the results of such a study and a first approach is provided on the organization of the space in the settlement and the stylistic modalities of the architectural group. Loma l'Ántigo is presented as an agglomerate village settled on top of an elevation that determines its internal configuration to the time that places it in a place highlighted in the landscape.
\end{abstract}

Keywords: Loma l'Ántigo, arquitecture, settlement, Late Period.

\section{Introducción}

A pesar de los numerosos vestigios de ocupaciones prehispánicas existentes en el valle del Cajón y de las tempranas exploraciones realizadas en la zona a fines del siglo XIX, el desarrollo de investigaciones sistemáticas en este valle fue discontinuo y fragmentado si lo comparamos con el de los valles aledaños. Sin embargo, en la década de los noventa esta situación comenzó a revertirse. Por un lado se iniciaron investigaciones tendientes a conocer la ocupación incaica en el valle (de Hoyos 1996a y b, 1999; de Hoyos y Williams 1994) y por 
otro, comenzaron estudios en la localidad de La Quebrada en el sur del valle del Cajón, con el objeto de comprender los modos de vida de las sociedades formativas. Estos trabajos generaron un programa a largo plazo, dedicado al estudio del pasado a través de distintas líneas de investigación (Scattolin y Gero 1999; Gero y Scattolin 2002; Izeta 2007; Scattolin et al 2007, 2009a y b; Bugliani 2008; Calo 2010; Cortés 2010, 2011; Pereyra Domingorena 2010).

En años recientes hemos retomado el interés en el Período Tardío iniciando investigaciones que tienen por objeto el estudio de las sociedades posteriores al primer milenio a través de sus prácticas y cultura material. En este contexto, se han emprendido estudios sistemáticos en el sitio Loma l'Ántigo, un asentamiento residencial que puede brindar información muy valiosa respecto de los modos de vida de tales poblaciones.

Loma l'Ántigo se localiza en el sector medio del valle del Cajón a 26 36' 57" latitud N y $66^{\circ} 20^{\prime} 57^{\prime \prime}$ longitud O (Figura 1). En este ámbito, el valle tiene unos $30 \mathrm{~km}$ de ancho, con una vertiente oriental muy abrupta sobre la cual se eleva la sierra del Cajón y una vertiente occidental más extendida, con planicie aluvional y elevaciones menores -morros y lomadasque se disponen en diferentes direcciones y son surcadas por los ríos que bajan desde las elevaciones del oeste. El sitio se emplaza sobre una de estas lomadas.

El objetivo de este trabajo es presentar los primeros resultados obtenidos a partir del relevamiento arquitectónico del asentamiento el cual permitió conocer la distribución y articulación de estructuras y rasgos construidos, las características del conjunto arquitectónico y las técnicas de construcción implementadas. Los resultados de tal registro proveen una primera aproximación sobre la organización del espacio en el asentamiento y exponen las modalidades estilísticas del conjunto arquitectónico.

\section{Investigaciones previas sobre el Tardío en el valle del Cajón}

Las primeras informaciones sobre la ocupación precolombina de este valle surgen a fines del siglo XIX. En 1893 H. Ten Kate, conservador de la Sección Antropología del Museo de La Plata, exploró el valle del Cajón como parte de una expedición que pretendía estudiar la arqueología Calchaquí (Ten Kate 1893). Sus observaciones incluyen costumbres y creencias de los pobladores del valle y hasta mediciones antropométricas; pero poco nos dicen de las evidencias materiales de quienes poblaron el valle en tiempos prehispánicos. En su descripción de vestigios arqueológicos hallados en los pueblos San Antonio y La Hoyada el autor menciona pircas rodeando espacios cuadrangulares que relaciona con corrales y rastrojos. Asimismo, en parajes circundantes señala la presencia de petroglifos y registra tumbas parcialmente destruidas. Resulta particularmente de interés para nuestro trabajo la descripción y registro de un 'pueblo antiguo', ubicado sobre una colina escarpada localizada entre los parajes Ovejería y Campo del Fraile (Ten Kate 1893). Se trata del primer relevamiento y publicación de datos que se hace del sitio Loma l'Ántigo del cual se ocupa el presente artículo.

En su descripción del asentamiento, Ten Kate contabilizó alrededor de 70 estructuras, realizó mediciones para conocer las diferencias de tamaño entre ellas y publicó un plano expeditivo mostrando la distribución de los recintos. En el epígrafe de la figura del plano, menciona este sitio como “Loma del Pueblito á Guasamayo” (Ten Kate 1893: 341). 
En las primeras décadas del siglo XX, Bruch y luego Schreiter exploraron el valle, pero se ocuparon de sitios en la margen izquierda del río Colorado, sobre la sierra del Cajón. Bruch (1911) visitó el sector sur y efectuó una descripción superficial del sitio Famabalasto. Por su parte, Schreiter (1919) realizó un recorrido desde Famabalasto hasta El Mishito y registró diferentes tipos de cementerios y entierros que luego utilizó para ilustrar su clasificación. En 1922 el Ing. Weiser concretó la cuarta expedición financiada por Muniz Barreto y visitó varios sitios en el valle del Cajón. Entre los inspeccionados menciona el pueblo viejo de Pampa Grande, un asentamiento pequeño sobre una loma que consideró "lindo, todavía bien conservado" (Weiser 1922: 40). De esta manera hace referencia al asentamiento objeto de nuestro estudio. La denominación que eligió se debe a que la loma se halla vecina a una planicie conocida como Pampa Grande. Weiser realizó un preciso levantamiento planimétrico de este poblado; más tarde este plano sería publicado por Raffino quien lo utilizó para ilustrar el trazado de damero irregular (Raffino 1988).

Durante las décadas siguientes el área no fue tema de investigaciones arqueológicas y sólo recién a mediados de los años '50 Cigliano centró sus investigaciones en la zona de Famabalasto situada sobre las sierras del Cajón y más al sur que Loma l'Ántigo (Cigliano 1956-57, 1958). Posteriormente María Delia Arena (1975) estudió las piezas cerámicas recuperadas por Weiser en Campo del Fraile. Asimismo, publicó los resultados obtenidos durante una campaña realizada al valle del Cajón por la División Arqueología del Museo de La Plata, en la cual buscó reconocer los sitios explorados por Weiser y ampliar la cantidad de sitios conocidos en el área. En Ovejería Chica visitó el poblado sobre la loma y también lo mencionó como Pampa Grande; allí realizó una descripción general de las construcciones en piedra y efectuó una recolección superficial del material cerámico.

En la década de los noventa, María de Hoyos inició un proyecto de investigación a fin de estudiar la ocupación incaica en los sectores medio y norte del valle del Cajón. Sus trabajos se concentraron en el conocimiento de los sistemas productivos estatales empleados por los incas en la región (de Hoyos y Williams 1994; de Hoyos 1999). Con este objetivo estudió el sistema de explotación agrícola incaico implementado en los alrededores de La Hoyada y San Antonio del Cajón. Asimismo realizó un amplio relevamiento de la zona y publicó una descripción detallada de las construcciones arqueológicas observadas sobre Loma del Antico, el mismo sitio que Weiser y Arena denominaron Pampa Grande (de Hoyos 1996b). Más recientemente esta autora inició trabajos aún en curso sobre el arte rupestre del valle (de Hoyos y Lanza 2000; de Hoyos et. al. 2000).

En resumen, las intervenciones en Loma l'Ántigo han sido escasas y esporádicas. Tempranamente se efectuaron planos más o menos detallados (Ten Kate 1893; Weiser 1922) y más tarde Arena y luego de Hoyos aportaron las primeras descripciones de la arquitectura del asentamiento. En estos casos se trató de información parcial dado que, como fue mencionado, el interés de estas investigadoras no estuvo puesto en este sitio, sino en otras problemáticas para las cuales el estudio de Loma l'Ántigo no era relevante.

A partir de 2009 iniciamos nuestras investigaciones en este poblado concentrado al que denominamos Loma l'Ántigo. Optamos por esta designación, respetando la manera en que los pobladores actuales y sus antecesores se referían al sitio, ya que consideramos pertinente el uso de topónimos locales. Además algunas de las nominaciones anteriores hacían referencia a ubicaciones confusas o a la topografía circundante, pero no a la elevación donde se localiza el sitio. 
A diferencia de los antecedentes mencionados nuestros trabajos ponen el foco en este asentamiento conglomerado ya que consideramos que es un promisorio ejemplo donde estudiar la dinámica entre personas, objetos e ideas en un contexto residencial del Período Tardío en el valle del Cajón, ámbito en el cual aún no se han estudiado los poblados tardíos en profundidad.

\section{Los relevamientos efectuados en Loma l'Ántigo}

Como primera intervención hemos realizado un relevamiento y descripción de la arquitectura del asentamiento con el fin de conocer su organización espacial, estilo arquitectónico y los elementos y técnicas constructivas utilizadas en su construcción.

El estudio combinó diferentes tipos de registro para obtener información de carácter cuantitativo y cualitativo. El relevamiento arquitectónico se realizó con brújula taquimétrica constatando la precisión del plano realizado por Weiser en 1922. Sobre el mismo se realizaron correcciones, ajustes y registro de nuevos datos. Además se cotejó la conservación de algunos rasgos observados en aquella época. El registro descriptivo tuvo en cuenta los materiales de construcción, el tipo, forma y dimensiones de las estructuras, las técnicas constructivas implementadas y la configuración espacial de los diferentes conjuntos discriminados. Asimismo se usó GPS para la georreferenciación de las estructuras y se realizó un registro fotográfico exhaustivo.

Para organizar el relevamiento y la descripción se utilizaron categorías propuestas por la Arqueología de la Arquitectura (Sánchez 1998). La forma de los elementos arquitectónicos y su organización fue definida a partir de la aplicación de estrategias del análisis formal: identificación y descripción de las construcciones, composición en materiales y técnicas constructivas, identificación de relaciones estratigráficas (Mañana Borrazás et al. 2002). Asimismo a través del análisis espacial se buscó conocer los factores organizativos considerando los modos de relaciones espaciales y las maneras y principios que los organizan (Ching 2002 [1982]). Por último, de modo preliminar, se exploraron aspectos sintácticos y perceptivos a partir del análisis del movimiento a través de las construcciones (circulación, permeabilidad).

Con los resultados de este relevamiento, que en esta primera etapa se presentan como descripción de los espacios construidos y su organización, se busca acceder a las formas de pensar y habitar el espacio en un sitio residencial tardío, atendiendo a cómo los lugares son diseñados para propiciar ciertas percepciones particulares y establecer determinadas relaciones sociales entre las personas que los ocupan.

\section{Configuración del sitio Loma l’Ántigo}

Loma l'Ántigo es un asentamiento residencial conglomerado ubicado a $2.700 \mathrm{msnm}$. Se emplaza sobre la cima más o menos aplanada de una loma. Esta localización permite la distribución extendida de las estructuras (Figura 2). Desde esta altura se obtiene un amplio panorama del valle. El conjunto arquitectónico se ve aislado por las características naturales de la elevación cuyas laderas son abruptas. Para acceder a la planicie superior hay que ascender unos $60 \mathrm{~m}$ con distinto grado de dificultad según el lateral por el que se trepe. 


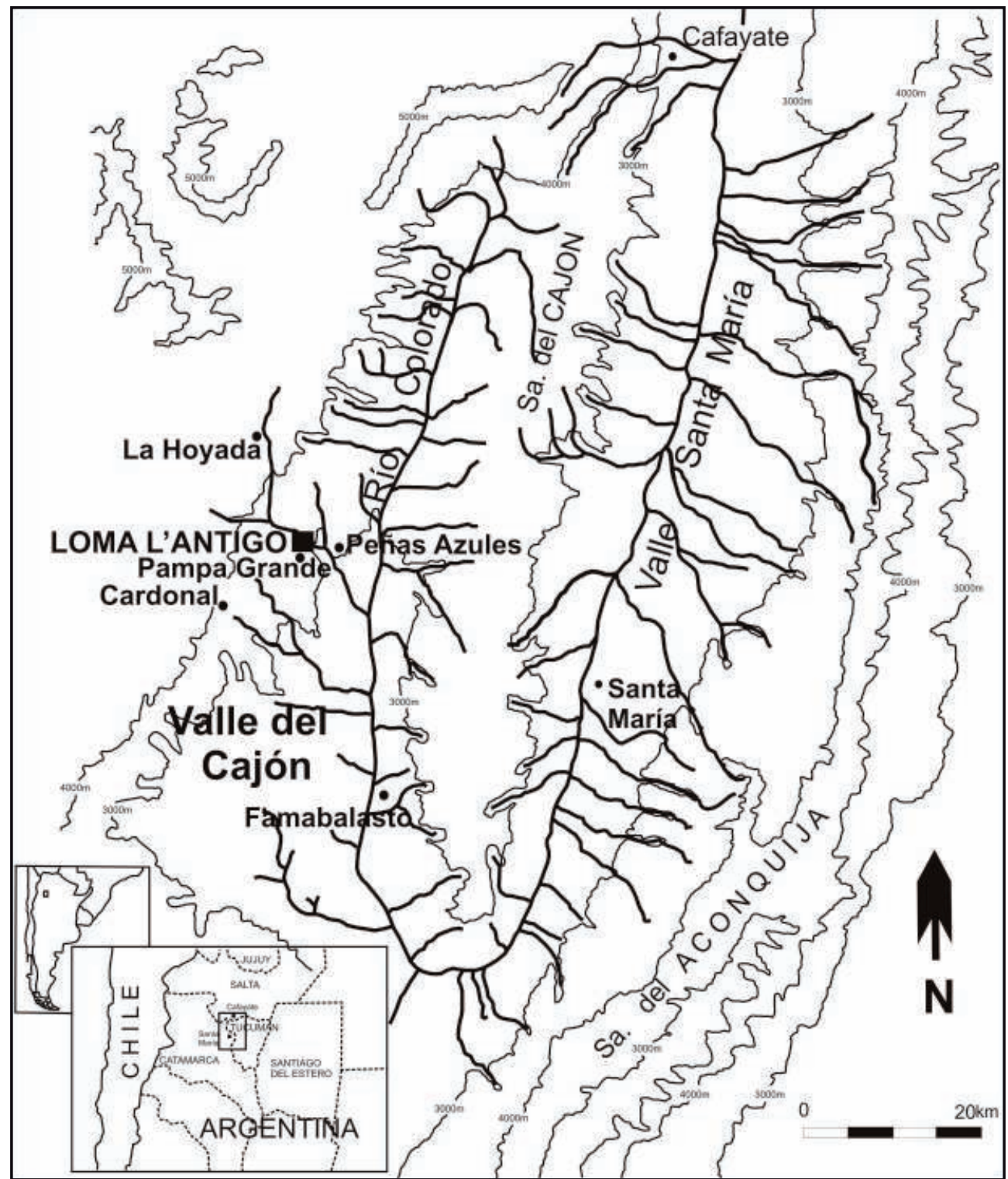

Figura 1. Localización del sitio Loma l'Ántigo.

$\mathrm{Al}$ pie de la loma, sobre el lado sur, se distribuyen una serie de recintos de piedra de planta rectangular y poligonal, cuyas dimensiones varían entre $100 \mathrm{~m}^{2}$ y $140 \mathrm{~m}^{2}$ de superficie y corresponden a campos agrícolas. Se contabilizaron 28 estructuras que se adosan compartiendo muros. En algunos casos se trata de paredes dobles con relleno. Las estructuras se organizan en dos conjuntos. A simple vista los recintos parecen haber sido construidos siguiendo un patrón irregular, pero esta disposición responde a la inclinación y morfología de la ladera sobre la cual se ha buscado distribuir los recintos a una cota uniforme. Dentro de algunas de estas estructuras se registraron pequeños muros de contención que no sobrepasan los $30 \mathrm{~cm}$ y se ubican paralelos entre si y a la pendiente. Los mismos están construidos con bloques de piedra en hilera simple. Más allá, pero adyacente a la loma, se 
extiende la planicie conocida como Pampa Grande donde Weiser (1922) señaló la presencia de sepulturas y actualmente se han constatado varios entierros huaqueados.

La loma se extiende en dirección SO-NE. El asentamiento sobre la elevación está constituido por 108 recintos que ocupan una extensión de 1,6 ha (Figura 3). Sobre la ladera norte, que es la más suave, han sido construidos dos muros paralelos entre sí, que siguen el nivel natural de la pendiente y están separados por ocho metros aproximadamente. El muro superior es doble con relleno de piedra y argamasa y tiene dos metros o más de ancho según los sectores. El muro inferior es simple y está constituido por bloques grandes ubicados en posición de contención de la pendiente. Sobre la ladera sur también aparecen algunas líneas de pared. Este lado es más abrupto y en el sector $\mathrm{SO}$ existen varios muros cortos de contención.

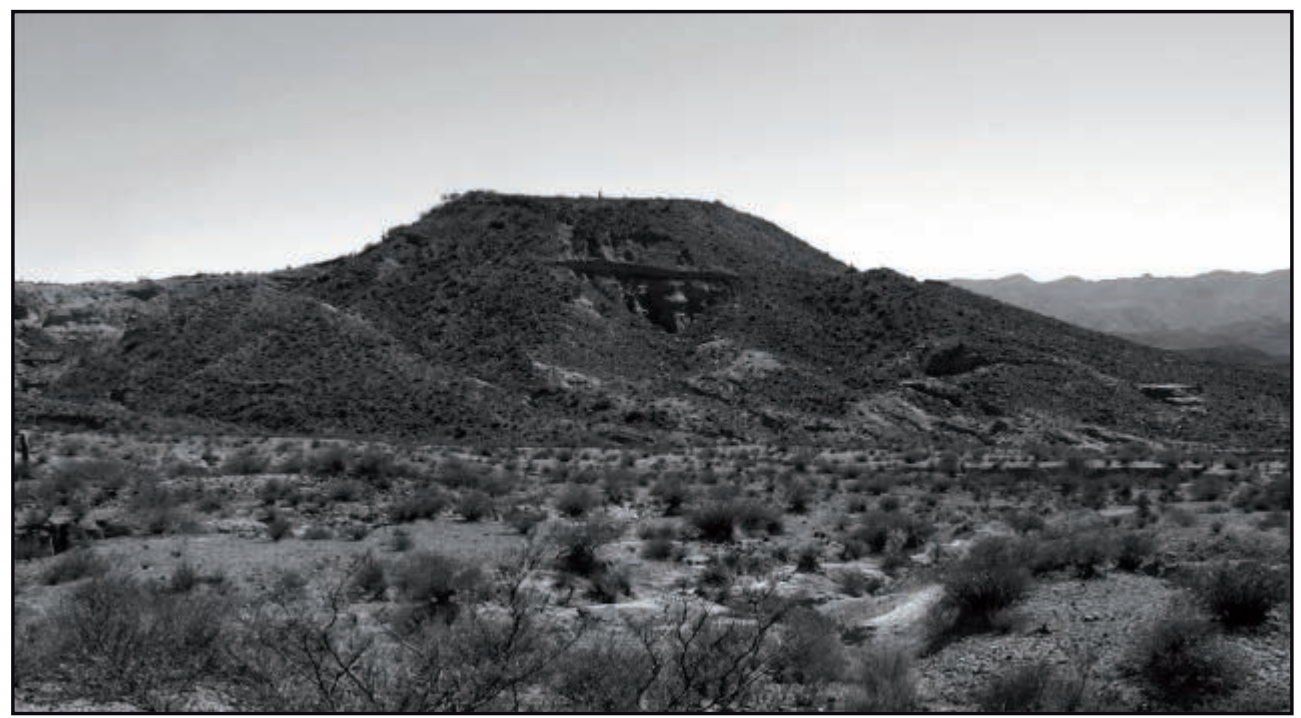

Figura 2. Vista de Loma l'Ántigo desde el sur. Nótese la forma aplanada de la cima de la loma donde se distribuye el poblado.

El estado de integridad o conservación de la arquitectura (Magadán 1988) es variado. Más de la mitad de los recintos presenta visibles los muros que delinean sus contornos (53 casos), en general las paredes no superan $\operatorname{los} 40 \mathrm{~cm}$ de alto aunque en ocasiones excepcionales se han registrado muros que conservan una altura de hasta $60 \mathrm{~cm}$ del piso actual (Figura 4e.). Otros 39 recintos presentan una integridad menor dado que algunos de sus muros o sectores de ellos están muy derrumbados o han desaparecido, haciendo poco identificable la forma de la habitación. Las 16 estructuras restantes presentan una baja integridad; casi no aparecen muros sobresalientes de la superficie del suelo y en algunos casos sólo son identificables por alineaciones de piedra ahora discontinuas.

\section{Técnicas constructivas y elementos arquitectónicos}

Se utilizó la piedra para la construcción de todos los recintos del poblado. No se han conservado remanentes de otros materiales que puedan haber sido empleados en las partes altas de los muros y en los techos. Como material de construcción se usaron bloques líticos, 


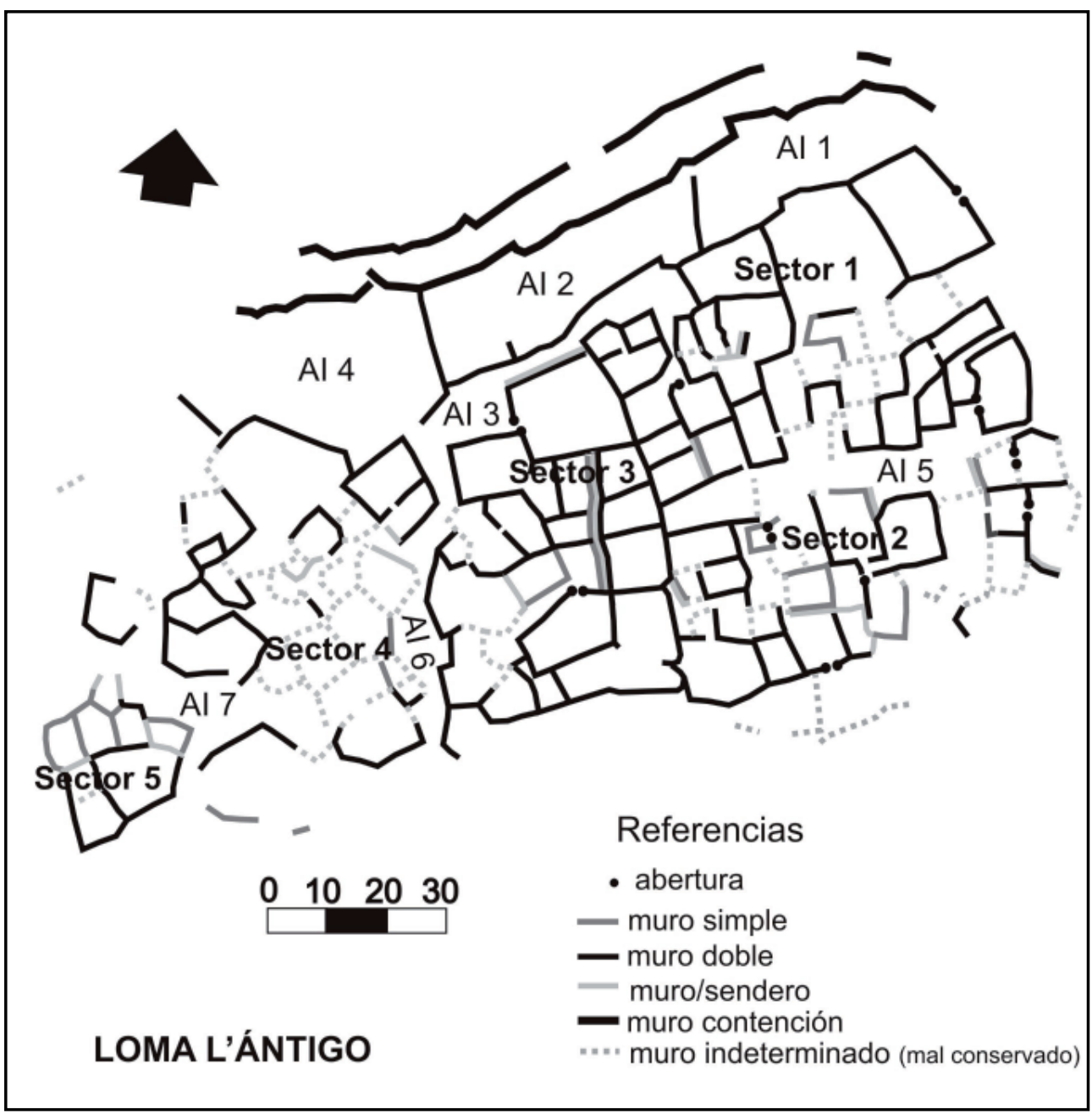

Figura 3. Plano de Loma l'Ántigo. Revisado y modificado a partir de Weiser 1922 y Raffino 1988.

muchos de ellos de formas redondeadas, los cuales son muy abundantes en los lechos de los ríos que circundan la loma.

El arreglo de los bloques en el paramento es variado, combinándose en un mismo lienzo la presencia de bloques grandes sostenidos por pequeñas cuñas y sectores con bloques pequeños y medianos (Figura 4a.).

El tipo de muro más frecuente es el muro doble con relleno formado por dos lienzos o mampuestos paralelos construidos con bloques de piedra apilados y con una cámara intermedia rellena de guijarros de menor tamaño, ripio y tierra (Figura 4c y d). Estas paredes suelen tener más de un metro de ancho, aunque en algunos casos han superado los dos metros.

También se utilizó el muro simple construido con bloques de piedra superpuestos. A veces ambos tipos de pared fueron combinados en la arquitectura de una misma habitación. En ciertas ocasiones los muros de dos estructuras contiguas deben haber actuado como vías 
de desplazamiento por las cuales circular entre recintos (Raffino 1988). Cuando observamos este rasgo, hemos denominado a estas paredes muro/sendero.

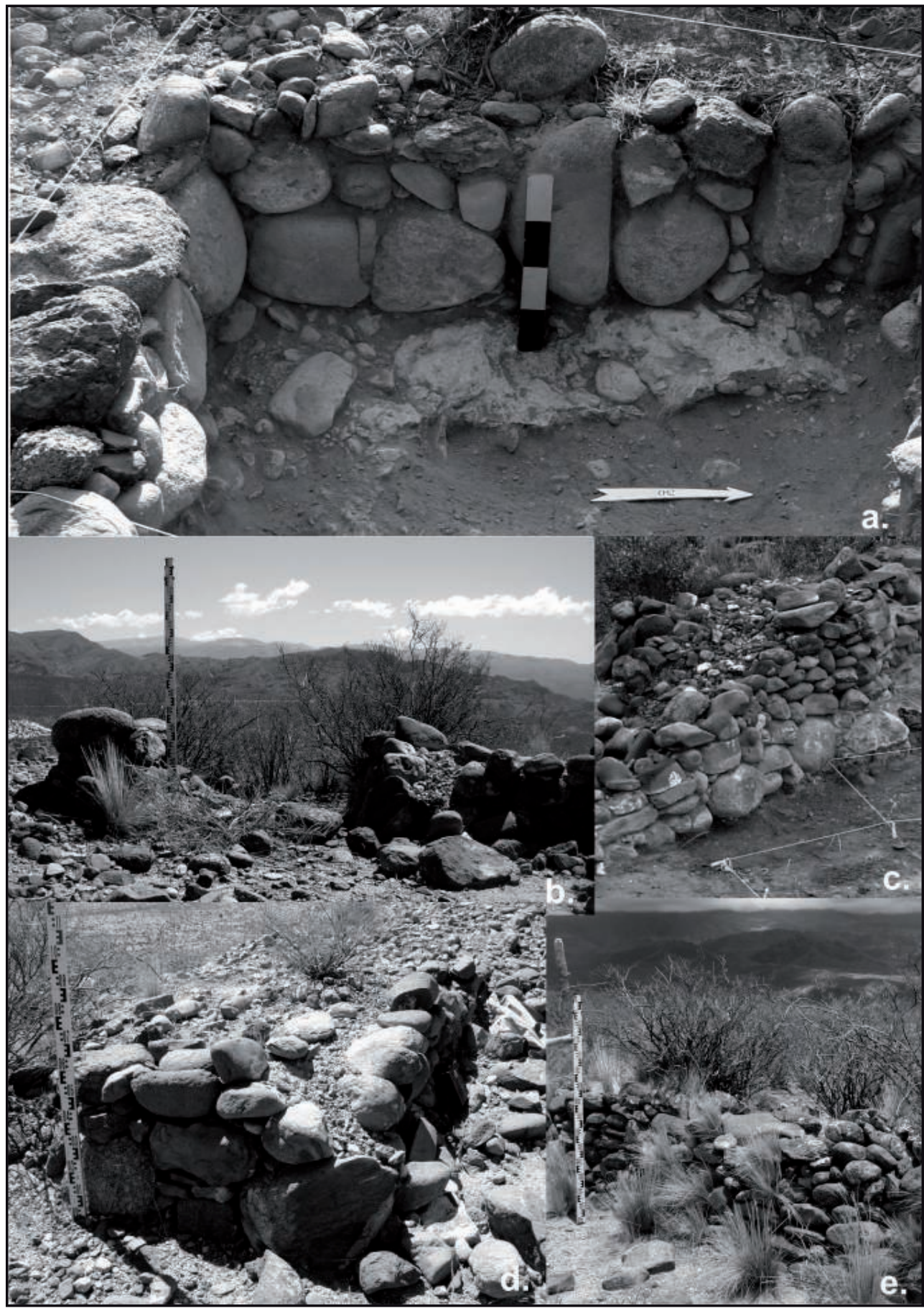

Figura 4. Rasgos arquitectónicos. a. detalle de muro, se observa la posición de piedras, cuñas y apoyo en cimientos. b. entrada a uno de los recintos. c. muro doble. d. entrada, nótese el ancho del muro doble que forma un pequeño pasillo. e. muro con buen estado de conservación. 
Asimismo, en algunos sectores donde se presenta un desnivel leve del terreno se observan algunos casos de muro simple empotrado revistiendo el perfil natural (Nastri 2001) y utilizado como contenedor de la pendiente.

\section{Configuración del asentamiento}

La forma de las estructuras otorga un primer instrumento para definir el espacio construido y delimitar interiores y exteriores. En este caso las plantas de todas las estructuras presentan formas poligonales. No existen recintos de planta circular. Los más frecuentes son los recintos de planta cuadrangular (50 casos) aunque existen también casos de formas rectangulares (19), trapezoidales (1) y de otras formas poligonales irregulares (38) (Figura 5).

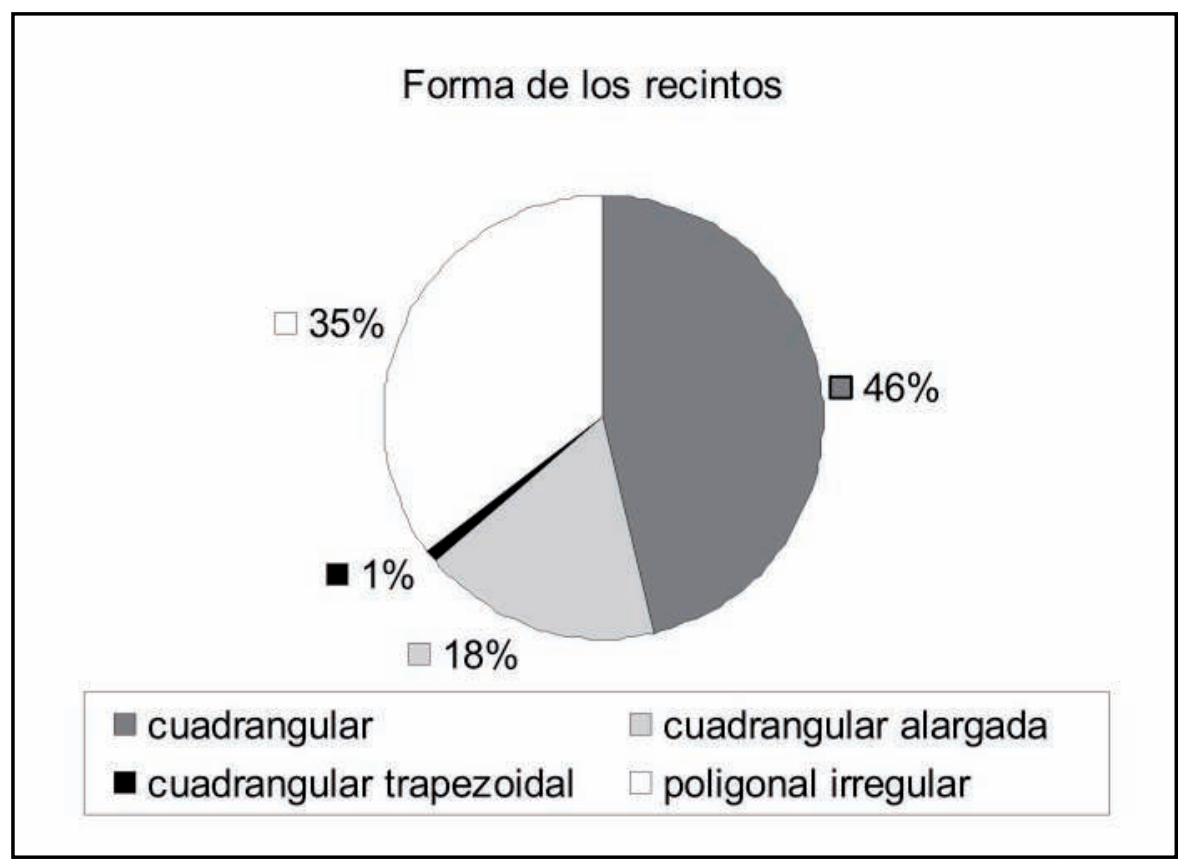

Figura 5. Representatividad de diferentes formas de recintos en Loma l'Ántigo.

Las estructuras se adosan compartiendo sus muros. Prima la continuidad (Ching 2002 [1982]) como modo de relación espacial entre recintos. En algunos casos las habitaciones se encuentran unidas por espacios comunicadores o conectores.

Si bien el plano del sitio muestra una distribución continua y aglomerada de los recintos, puede observarse la presencia de espacios intermedios que permiten sectorizar el conjunto mayor en términos analíticos. En esta distribución interviene como condicionante la topografía del terreno aunque no se descartan implicancias organizativas de la dinámica de crecimiento y ordenamiento del poblado. Estos espacios intermedios de formas poligonales y de mayores dimensiones que el común de las estructuras han sido denominados Áreas Intermedias (AI). Se trata en su mayoría de espacios de forma alargada ubicados entre los conjuntos de recintos. En algunos casos la separación entre dos conjuntos de estructuras está dada por un desnivel de dos o tres metros y el espacio intermedio es el que salva esta diferencia altitudinal. 
Las Áreas Intermedias 1, 2 y 4 se encuentran adyacentes al muro de contención superior del lado norte, contiguas entre sí y separadas por anchos muros dobles. Las otras Áreas Intermedias $(3,5,6$ y 7$)$ se ubican entre recintos y permiten distinguir sectores más o menos diferenciables (Figura 3).

Los Sectores 1 y 2 se encuentran separados por el AI5. A su vez estos sectores se separan del 3 delimitado por un ancho muro doble compartido por varias estructuras alineadas. El Sector 4 está separado del 3 por el AI6 que representa un desnivel importante del terreno. En un nivel inferior y separado del Sector 4 por AI7 se encuentra el Sector 5.

\section{Consideraciones finales}

Loma l'Ántigo es un poblado residencial asentado sobre un morro que determina su configuración, al tiempo que lo emplaza en un sitio destacado del paisaje. La fisonomía del asentamiento es la de un poblado aglomerado y su disposición está definida por la forma alargada del escaso espacio amesetado en la cima de la loma. La distribución de los recintos es extendida en sentido SE-NO y se prolonga sobre toda la superficie aplanada.

El asentamiento se configuró a partir de la acumulación de estructuras dispuestas adosadas unas a otras y separadas por espacios poco formalizados. Excepto por estos espacios que debieron ser abiertos y que se localizan entre las estructuras no se advierte fragmentación ni sectores jerarquizados en términos arquitectónicos.

Pueden advertirse dos tipos básicos de organización espacial (Ching 2002 [1982]). En el conjunto de habitaciones concentrado en el lado este de la loma (conformado por los sectores denominados 1, 2 y 3) sobresale la ordenación de tipo lineal, donde las estructuras están organizadas por un eje a lo largo del cual las unidades de tamaños más o menos similares se repiten. En la mayoría de los casos los recintos se vinculan directamente compartiendo paredes rectas y quedan de esta forma alineados. La otra mitad, que ocupa el lado oeste (Sectores 4 y 5), presenta una distribución menos regular, con estructuras que se adosan y articulan de manera no alineada. Predomina en esta parte una organización de tipo agrupada donde las unidades se concentran solamente por relaciones de proximidad. Estos sectores carecen de regularidad geométrica; aún no queda claro cuál es la solución de esta reunión particular. Además, los tamaños de los recintos son menos homogéneos.

De acuerdo a este primer relevamiento, las diferencias observadas en los modos de organización espacial de las estructuras en distintos sectores no se corresponden con la utilización diferencial de materiales ni de técnicas constructivas.

Por otra parte, las estructuras se encuentran muy aglomeradas y no se observan claros senderos de circulación entre las unidades. Se presume que los anchos muros de gran cantidad de las estructuras han servido como lugares de circulación. Asimismo, los espacios que denominamos Áreas Intermedias deben haber funcionado como distribuidores y áreas de tránsito, ya que su sentido longitudinal y paralelo a varias estructuras, induce el modo de aproximación a la unidad y el sentido de circulación.

Ha sido dificultoso advertir los lugares de ingreso y egreso a los recintos. Sólo en ocho estructuras fue posible identificar las aberturas gracias a su buen estado de conservación. Se trata de discontinuidades en los muros, que en el caso de los de tipo doble, han sido 
interrumpidos por un mampuesto que les da el corte. Hemos observado también, que el ancho de las paredes dobles, que como dijimos puede superar el metro, genera un pequeño pasillo de ingreso/egreso en estas aberturas (Figura 4d). En los casos registrados las conexiones son variadas, facilitando el acceso entre dos o tres recintos o entre una habitación y un espacio abierto.

En líneas generales el asentamiento presenta las características que menciona Raffino para definir un trazado de damero irregular: ausencia de estandarización de las unidades residenciales, resolución de la distribución a partir de agrupamiento de recintos iguales o desiguales intercomunicados, inexistencia de formalización generalizada de las estructuras, aspecto anárquico de la instalación que da idea de crecimiento espontáneo al menos en los inicios y carácter estratégico, posiblemente defensivo, inferido por el emplazamiento sobre terrenos ríspidos sobre la cima (Raffino 1888: 193). Por su parte, Arena resaltó que el sitio poseía un emplazamiento estratégico y postuló que se trataba de un lugar de refugio, ocupado de manera temporaria, lo que infería a partir de la falta de basureros, acumulaciones superficiales y morteros entre otras evidencias (Arena 1975: 48-49). Coincidimos en el carácter estratégico que tiene este emplazamiento en el paisaje, con una extensa visibilidad hacia todos los sectores circundantes y con disponibilidad de recursos hídricos muy próximos.

Sin embargo, si bien es cierto que el material arqueológico hallado en superficie es escaso, la manera en que están construidos las estructuras y demás rasgos arquitectónicos no parece mostrar una estrategia expeditiva o temporaria. Los trabajos de campo en curso han permitido recuperar diversos elementos (material cerámico, óseo, lítico) y conocer rasgos (cimientos, hoyos cavados en la roca de base) en el contexto de un piso de ocupación bien conservado ${ }^{1}$. Su estudio exhaustivo nos permitirá acceder a las diferentes actividades desarrolladas en un recinto del conjunto habitacional, pero de manera preliminar puede adelantarse que en esta estructura (E93) se registraron actividades de combustión y otras de procesamiento y posiblemente consumo de alimentos de origen animal.

Por su parte, los sondeos efectuados en diversos sectores de Loma l'Ántigo han permitido registrar diferencias en la composición de la estratigrafía debido a su localización. Así hemos podido constatar distinciones de acuerdo a la topografía sobre la que se asientan las estructuras.

Aún es necesario avanzar en la determinación de la dimensión temporal contenida en la construcción y uso de este asentamiento. Los rasgos arquitectónicos registrados así como la forma en que se organizaron las estructuras en Loma l'Ántigo son comparables con las maneras de construir observadas en otros poblados del Período Tardío. Asimismo, las asociaciones estilísticas en los materiales cerámicos permiten ubicar la ocupación del sitio dentro de este lapso. Contamos con partes de ollas ordinarias peinadas y con base en pie de compotera junto con fragmentos asignables al Santa María Bicolor (Negro sobre Blanco) y otros tiestos pintados en Negro sobre Rojo. El análisis de estos materiales y de toda la información recuperada en los trabajos de campo, junto con la continuidad de las investigaciones en curso, generará nuevas evidencias que nos permitan conocer en profundidad este asentamiento y las experiencias de quienes lo habitaron.

\section{Agradecimientos}

Agradezco la hospitalidad de Mercedes Pachado, José Pachado, Ana Gutiérrez y toda la familia durante nuestras estadías en Ovejería Chica. Asimismo quiero agradecer la colaboración de Leticia Cortés, Ma. Clara Quintero y María Pérez en los trabajos de campo en Loma l'Ántigo. De gran valor fueron los comentarios realizados a una 
primera versión de este trabajo por la Dra. Laura Quiroga. Estas investigaciones han sido sostenidas con fondos del proyecto PICT 06-1244. “Estilos cerámicos y prácticas sociales posteriores al Formativo en el valle del Cajón (Pcia de Catamarca)".

\section{Notas}

1. Aún se encuentran en proceso de estudio los datos y materiales arqueológicos recuperados en sondeos practicados en diferentes sectores del asentamiento y la excavación en área de una de las estructuras (E93).

\section{Bibliografía citada}

Arena, M.D.

1975. Arqueología del Campo del Fraile y aledaños (Valle del Cajón, Depto. Santa María, Catamarca). En: Actas y trabajos del Primer Congreso de Arqueología Argentina. pp. 43-96. Buenos Aires.

Bruch, C.

1911. Exploraciones arqueológicas en las provincias de Tucumán y Catamarca. Revista del Museo de La Plata, 19. Biblioteca Centenaria.

Bugliani, M.F.

2008. Consumo y representación en el sur de los valles Calchaquíes (Noroeste argentino): Los conjuntos cerámicos de las aldeas del primer milenio A.D. BAR (British Archaeological Reports), International Series, S1774. Oxford.

Calo, C. M.

2010. Plantas útiles y prácticas cotidianas entre los aldeanos al sur de los Valles Calchaquíes. Tesis Doctoral inédita. Facultad de Ciencias Naturales y Museo. Universidad Nacional de La Plata.

Ching, F.

2002 [1982]. Arquitectura. Forma, espacio y orden. Ediciones G. Gili. México.

Cigliano, E. M.

1956-57. Investigaciones arqueológicas en la zona de Famabalasto (Prov. de Catamarca). Runa VIII 2da. parte: 241-269.

Cigliano, E. M.

1958. Arqueología de la zona de Famabalasto, Departamento de Santa María, Catamarca. Extracto de la Revista del Museo de La Plata (Nueva Serie), Sección Antropología, Tomo V: 29-122.

Cortés, L. I.

2010. Cuerpos en contraste: reflexiones sobre el tratamiento de los difuntos en dos entierros de 3000 años (valle del Cajón, Noroeste argentino). Revista del Museo de Antropología 3: 5-12.

Cortés, L. I.

2011. Paisaje funerario al sur del valle del Cajón: cuerpos, contextos y trayectorias históricas. Tesis Doctoral inédita. Facultad de Filosofía y Letras. Universidad de Buenos Aires. 
de Hoyos, M.

1996a. La Hoyada. Un enclave de producción agrícola en el valle del Cajón. Provincia de Catamarca. Actas y Memorias del XI Congreso Nacional de Arqueología Argentina (13 Parte). Revista del Museo de Historia Natural de San Rafael XXV (1/4): 273-293. San Rafael.

de Hoyos, $\mathrm{M}$.

1996b. Centros de producción agrícola en el Valle del Cajón. Actas y Memorias del XI Congreso Nacional de Arqueología Argentina (13 ${ }^{\circ}$ Parte). Revista del Museo de Historia Natural de San Rafael XXIV (1/4): 295-317. San Rafael.

de Hoyos, M.

1999. Asentamiento inka en San Antonio del Cajón (Dpto. de Santa María-Catamarca). Actas del XII Congreso Nacional de Arqueología Argentina. Tomo I: 194-198. La Plata.

de Hoyos, M. y M. Lanza

2000. Arte rupestre en San Antonio del Cajón. Provincia de Catamarca. Relaciones de la Sociedad Argentina de Antropología XXV: 119-144.

de Hoyos, M, M. Lanza y L. Horlent 2000. Bloques con grabados en San Antonio del Cajón, Catamarca. Arte en las rocas (ed. por .M. Podestá, y M. de Hoyos), pp. 83-93. Sociedad Argentina de Antropología y Asociación Amigos del INAPL. Buenos Aires.

de Hoyos y V. Williams

1994. Un patrón de asentamiento estatal para propósitos especiales. Actas y Memorias del XI Congreso Nacional de Arqueología Argentina (Resúmenes). Revista del Museo de Historia Natural de San Rafael (Mendoza). Tomo XIII (1/4): 196-199. San Rafael.

Gero, J. M. y M. C. Scattolin

2002. Beyond complementary and hierarchy: new definitions for archaeological gender relations. In Pursuit of Gender: Worldwide Archaeological Approaches (ed. por S.M Nelson y M. Rosen-Ayalon), pp. 155-171. Walnut Creek, Altamira Press.

Izeta, A. D.

2007. Zooarqueología del sur de los valles Calchaquies (Provincias de Catamarca y Tucumán, República Argentina): Análisis de conjuntos faunísticos del primer milenio A.D. B.A.R. (British Archaeological Reports), International Series S1612. Oxford.

Magadán, M.

1988. Propuesta de una ficha para el relevamiento de restos arquitectónicos en sitios prehispánicos. Publicación $n^{\circ}$ 8. Arqueología Urbana. Instituto de Arte Americano e Investigaciones Estéticas J. Buschiazzo. Facultad de Arquitectura y Urbanismo. Universidad de Buenos Aires.

Mañana Borrazás, P., R. Blanco Rotea y X. Ayán Vila.

2002. Arqueotectura1: bases teórico metodológicas para una arqueología de la arquitectura. TAPA Traballos de Arqueología e Patrimonio 25. Universidade de Santiago de Compostela. Santiago de Compostela. 
Nastri, J.

2001. La arquitectura aborigen de la piedra y la montaña (Noroeste Argentino, siglos XI a XVII). Anales del Museo de América 9: 141-163.

Pereyra Domingorena, L.

2010. Manufacturas alfareras de las sociedades aldeanas del primer milenio d.C. al sur de los valles Calchaquíes. Tesis Doctoral inédita. Facultad de Filosofía y Letras, Universidad de Buenos Aires, Buenos Aires.

Raffino, R.

1988. Poblaciones indígenas en Argentina. Editorial TEA. Buenos Aires.

Sánchez, J.

1998. La arqueología de la arquitectura. Aplicación de nuevos modelos de análisis a estructuras de la Alta Andalucía en Época Ibérica. Trabajos de Prehistoria 55 (2): 89-109.

Scattolin M. C y J. M. Gero

1999. Consideraciones sobre fechados radiocarbónicos de Yutopián, Catamarca, Argentina. Actas del XII Congreso Nacional de Arqueología Argentina. Tomo III: 352-357. La Plata.

Scattolin , M. C.; L. Pereyra Domingorena, L. I. Cortés, M. F. Bugliani, C. M. Calo, A. D. Izeta y M. Lazzari

2007. Cardonal: una aldea formativa entre los territorios de valles y puna. Cuadernos UNJu 32: $211-225$.

Scattolin, M. C., M. F. Bugliani, L. I. Cortés, C. M. Calo, L. Pereyra Domingorena y A. D. Izeta

2009a. Pequeños mundos: hábitat, maneras de hacer y afinidades en aldeas del valle del Cajón, Catamarca. Relaciones de la Sociedad Argentina de Antropología 34: 251-274.

Scattolin, M. C.; L. I. Cortés, M. F. Bugliani, C. M. Calo, L. Pereyra Domingorena, A. D. Izeta y M. Lazzari

2009b. Built landscapes of everyday life: a house in an early agricultural village of northwestern Argentina. World Archaeology 41 (3): 396-414.

Schreiter, R.

1919. Distintas clases de sepulturas antiguas observadas en los Valles Calchaquíes. Ueberreicht com Verfasser. Sonderabdruck aus Zeitschrift des Deutschen Wissenchaftlichen Vereins zur Kultur-und Landeskcnde Argentiniens. Buenos Aires.

Ten Kate, H. F.

1893. Rapport sommaire sur une excursion archéologique dans les provinces de Catamarca, de Tucumán et Salta. Revista del Museo de La Plata, V: 331-384.

Weiser, W.

1922. Diario de la 4ta Expedición Benjamin Muniz Barreto. Departamento Arqueología. Museo de La Plata. MS. 